OPEN ACCESS

Edited by:

Dong Ho Park

Kyungpook National University

Hospital, South Korea

Reviewed by:

Yong Koo Kang

Kyungpook National University,

South Korea

Min-Woo Lee,

Konyang University, South Korea

*Correspondence:

Chun-Ju Lin

doctoraga@gmail.com

Specialty section: This article was submitted to

Ophthalmology,

a section of the journal

Frontiers in Medicine

Received: 15 February 2021 Accepted: 06 April 2021

Published: 03 May 2021

Citation:

Lai C-T, Hsieh Y-T, Lin C-J, Wang J-K, Lin $C-Y$, Hsia N-Y, Bair H, Chen H-S,

Chiu C-Y and Weng S-W (2021) Age,

Initial Central Retinal Thickness, and

OCT Biomarkers Have an Influence on the Outcome of Diabetic Macular Edema Treated With RanibizumabTri-center 12-Month Treat-and-Extend

Study. Front. Med. 8:668107. doi: 10.3389/fmed.2021.668107

\section{Age, Initial Central Retinal Thickness, and OCT Biomarkers Have an Influence on the Outcome of Diabetic Macular Edema Treated With Ranibizumab- Tri-center 12-Month Treat-and-Extend Study}

Chun-Ting Lai ${ }^{1}$, Yi-Ting Hsieh ${ }^{2}$, Chun-Ju Lin ${ }^{1,3,4 *}$, Jia-Kang Wang ${ }^{5,6}$, Chih-Ying Lin ${ }^{1,3}$, Ning-Yi Hsia ${ }^{1,3}$, Henry Bair ${ }^{1}$, Huan-Sheng Chen ${ }^{7}$, Chiung-Yi Chiu ${ }^{5}$ and Shao-Wei Weng ${ }^{5}$

${ }^{1}$ Department of Ophthalmology, China Medical University Hospital, China Medical University, Taichung, Taiwan, ${ }^{2}$ Department of Ophthalmology, National Taiwan University Hospital, Taipei, Taiwan, ${ }^{3}$ School of Medicine, College of Medicine, China Medical University, Taichung, Taiwan, ${ }^{4}$ Department of Optometry, Asia University, Taichung, Taiwan, ${ }^{5}$ Department of Ophthalmology, Far Eastern Memorial Hospital, Taipei, Taiwan, ${ }^{6}$ Department of Electrical Engineering, Yuan Ze University, Taoyuan City, Taiwan, ${ }^{7}$ An-Shin Dialysis Center, NephroCare Ltd., Fresenius Medical Care, Taichung, Taiwan

Objective: We report the tri-center 1-year outcomes of a treat-and-extend (T\&E) regimen in four-week intervals with ranibizumab for diabetic macular edema (DME).

Methods: In this retrospective study, all eyes received 3 monthly loading injections of $0.5 \mathrm{mg}$ ranibizumab, followed by a T\&E regimen for DME. Regression models were used to evaluate the associating factors for visual and anatomical outcomes.

Results: Ninety one eyes from 64 patients were enrolled. Mean LogMAR best-corrected visual acuity (BCVA) improved from 0.58 at baseline to 0.36 at month 12 and mean central retinal thickness (CRT) decreased from $411 \mu \mathrm{m}$ at baseline to $290 \mu \mathrm{m}$ at month 12. Younger age and eyes having thinner baseline CRT, with ellipsoid zone disruption (EZD), and without epiretinal membrane (ERM) were associated with better final CRT. Moreover, eyes with thicker baseline CRT tend to receive more injections. Among the parameters, only having ERM or EZD was associated with significant BCVA recovery.

Conclusions: A T\&E regimen with ranibizumab by 4-week intervals is effective in improving BCVA and reducing CRT with efficacy notable starting from the third month. Clinical parameters including age, initial CRT, and presence of ERM or EZD significantly influenced therapeutic outcomes. Moreover, the presence of ERM should not preclude DME patients from receiving anti-VEGF therapy. Future studies with larger cohorts are warranted.

Keywords: age, central retinal thickness, diabetic macular edema, ОCT biomarkers, ranibizumab, treat-and-extend regimen 5 


\section{INTRODUCTION}

Diabetic retinopathy (DR) affects an estimated one in three people with diabetes mellitus (DM) (1) and causes severe visual impairment. Diabetic macular edema (DME), a common complication of DR, can present in both non-proliferative diabetic retinopathy (NPDR) and proliferative diabetic retinopathy (PDR) (2).

DME is pathologically linked to the disruption of the blood retinal barrier. In the hypoxic microenvironment of DR, vascular endothelial growth factor (VEGF) increases capillary permeability and breaks down blood retinal barrier (3). Ranibizumab (Lucentis, Genentech Inc., South San Francisco, CA), an anti-angiogenic agent, has revolutionized the treatment of DME. The RISE and RIDE phase II trial showed that $44.8 \%$ of patients gain more than 15 letters in vision after monthly injections of $0.3 \mathrm{mg}$ ranibizumab (4). The success of ranibizumab over intravitreal steroid and photocoagulation monotherapy has also been established in the literatures $(5,6)$.

Nonetheless, monthly injections of ranibizumab is impractical as the cost of anti-VEGF agents and the requirement of frequent clinic visits be barriers to patient compliance to regimen (7). The TREX-DME study demonstrated that treat and extend (T\&E) dosing was comparable with monthly dosing and allows for incremental increases in treatment intervals by 2 weeks. This resulted in less frequent injections and less expenditure (8). Therefore, T\&E dosing with 4-week intervals may be more practical in terms of reducing treatment burden.

Despite robust findings from clinical trials, around half of eyes do not fully respond to anti-VEGF (9), and further exploration of prognostic factors associated with better visual outcomes is warranted. Age, HbAlc status, central retinal thickness (CRT) have been investigated but to mixed results (10-13). Moreover, little is known regarding how optical coherence tomography (OCT) biomarkers including epiretinal membrane (ERM) and ellipsoid zone disruption (EZD) affect the resolution of macular edema and final vision, and its implication for therapeutic strategy.

This tri-center 12 month study aims to investigate the efficacy of ranibizumab on DME following a regimen of 3 monthly loading injections plus 4 -week T\&E therapeutic intervals. To understand favorable factors for functional and anatomical visual outcomes, we assessed clinical parameters of patients with different therapeutic responses.

\section{MATERIALS AND METHODS}

\section{Subjects}

This retrospective study was conducted from 2017 to 2019 at the Department of Ophthalmology of three tertiary centers in Taiwan (China Medical University Hospital, National Taiwan University Hospital, and Far Eastern Memorial Hospital). We reviewed subjects with either type I or type II DM and a concomitant DR diagnosis. DME diagnosis was made according to features of exudates and macular thickening on fundus and OCT exam. CRT was calculated as the average thickness of the central
$1,000 \mu \mathrm{m}$ diameter area (14) with spectral domain OCT (SDOCT) device (Heidelberg Engineering, Heidelberg, Germany). Among the OCT biomarkers, EZD is defined as having any discontinuity of the second hyper-reflective layer of fovea on OCT. The shadowing effect of cysts and retinal vessels was not regarded as part of the EZD (15).

Inclusion criteria for receiving ranibizumab were as follows: eyes having Snellen best-corrected visual acuity (BCVA) between $20 / 400$ and 20/40, CRT on OCT being $>300 \mu \mathrm{m}$ at the initial presentation, and eyes demonstrating late onset hyperfluorescence typical of macular leakage on fluorescence angiography (FA). Exclusion criteria involves having macular edema of non-diabetic causes, a history of vitrectomy and laser photocoagulation 3 months prior to study entry. Among PDR patients, subjects who were treated with additional laser photocoagulation during the study period were excluded. OCT images of poor quality were excluded as well.

The study protocol was approved by the Institutional Review Board and informed consent was obtained from all participants. The study complies with the tenets of the Declaration of Helsinki.

\section{Study Design and Statistical Analysis}

All patients received 3 monthly loading injections of $0.5 \mathrm{mg}$ ranibizumab, followed by a T\&E algorithm in which the treatment intervals were increased by 4 weeks after reaching a stable BCVA status and a CRT $<300 \mu \mathrm{m}$. Ranibizumab injections interval were reduced by 4 weeks if the individual had vision loss due to DME recurrence. DME recurrence was defined as having CRT $>300 \mu \mathrm{m}$. If there was no recurrence, patients were allowed to extend their clinical visit and injection one more month due to personal reasons (Figure 1). All subjects were followed for at least 12 months.

Primary outcomes included variations in BCVA and CRT after 12 months of treatment. Secondary outcomes were the univariate analysis and multivariate logistic regression analysis of the biomarkers that predicted better BCVA outcomes in DME. We applied Chi-square for the univariate analysis of categorical variable and ANOVA for numerical variable. In the multivariate logistic regression analysis, eventual CRT, BCVA changes and injection times were dependent variables. Baseline parameters such as age, DR staging and OCT biomarkers were independent variables. Statistical analysis was conducted with Statistical Package for the Social Sciences (SPSS) version 22.0 for Windows.

\section{RESULTS}

Ninety-one eyes of 64 patients with DME were enrolled. Thirty five $(54.7 \%)$ males and $29(45.3 \%)$ females were included. The baseline $\mathrm{HbA1C}$ was $7.44 \pm 1.02 \%$. There were 4 eyes of mild NPDR, 14 of moderate NPDR, 28 of severe NPDR, 6 of PDR, and 39 of eyes with PDR that had received PRP (Table 1). The majority of DR staging was severe NPDR and PDR.

The mean injection number was $7.67 \pm 2.09$ (5-12; 95\% confidence interval) with $71.21 \%$ eyes received five to eight injections. The majority of cases needed eight injections. The proportion of eyes with BCVA improvement had gradually 


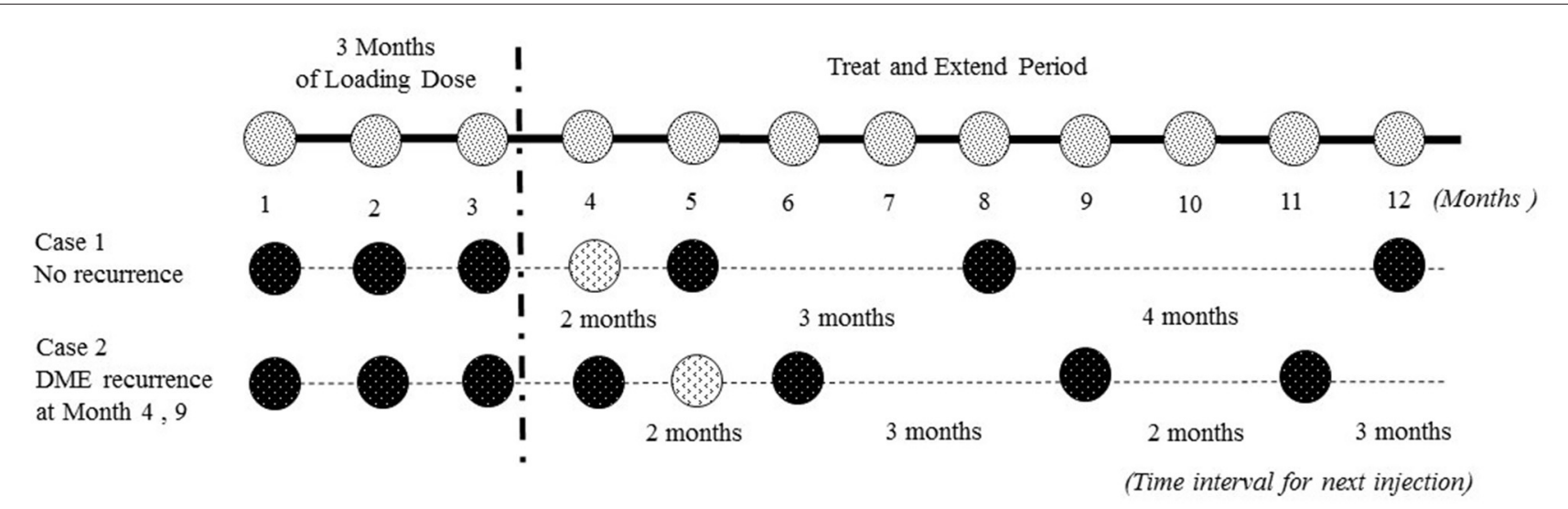

FIGURE 1 | Illustrated the treat and extend (T\&E) dosing algorithm in our real world study to determine treatment intervals. In this study, there were 3 monthly injections of ranibizumab, and injection intervals were extended by 4 weeks if there was no DME recurrence. DME recurrence was defined as having central retinal thickness $>300 \mu \mathrm{m}$. If there was no recurrence, patients were allowed to extend their clinical visit and injection one more month due to personal reasons. BCVA, best-corrected visual acuity; DME, diabetic macular edema.

TABLE 1 | Demographic data.

\begin{tabular}{lc}
\hline Baseline characteristics & All patients $(\mathbf{N}=64)$, Eyes $(\boldsymbol{n}=\mathbf{9 1})$ \\
\hline Age & $60.31 \pm 10.75$ \\
Gender & \\
$\quad$ Female & $29 / 64(45.3 \%)$ \\
HbA1C (\%) & $7.44 \% \pm 1.02 \%$ \\
CRT ( $\mu$ m) & $411.30 \pm 114.61$ \\
LogMAR & $0.58 \pm 0.36$ \\
DR staging & \\
Mild NPDR & \\
Moderate NPDR & $4 / 91(4.40 \%)$ \\
Severe NPDR & $14 / 91(15.4 \%)$ \\
PDR & $28 / 91(30.8 \%)$ \\
PDR with PRP & $6 / 91(6.59 \%)$ \\
IRC & $39 / 91(42.9 \%)$ \\
HE & $71 / 91(78.0 \%)$ \\
DRIL & $70 / 91(76.9 \%)$ \\
EZD & $31 / 91(34.1 \%)$ \\
ERM & $26 / 91(28.6 \%)$ \\
SRF & $23 / 91(25.3 \%)$ \\
\hline
\end{tabular}

N, number; CRT, Central Retinal Thickness; DR, Diabetic Retinopathy; NPDR, Non Proliferative Diabetic Retinopathy; PDR, Proliferative Diabetic Retinopathy; PRP, Panretinal Photocoagulation; IRC, Intra-Retinal Cyst; HE, Hard Exudate; DRIL, Disorganization of Retinal Inner Layers; EZD, Ellipsoid Zone Disruption; ERM, Epiretinal Membrane; SRF, Subretinal Fluid.

increased from $58.2 \%$ since Month three and reached $72.5 \%$ at month 12. The mean LogMAR BCVA improved significantly from 0.58 at baseline to 0.36 in month 12 (Figure 2). The mean CRT decreased significantly from $411.3 \mu \mathrm{m}$ at baseline to $289.8 \mathrm{~m}$ in month 12 (Figure 3). In both Figures 2, 3, all the $p$-values shown were compared to the baseline LogMAR and baseline CRT, respectively.

Next, we performed inter-cohort univariate and multivariate analysis. Patients were further classified according to final CRT thickness ( $>$ or $<300 \mu \mathrm{m}$ ), the final change in BCVA (with or without BCVA improvements) and total injection times (more or fewer than six injections) (Table 2). In the inter-cohort analysis of final CRT $>$ or $<300 \mu \mathrm{m}$, eyes of younger age and eyes having thinner baseline CRT, with EZD, and without ERM were associated with better final CRT $(<300 \mu \mathrm{m})$ (Table 2). This correlation were also confirmed in multivariate analysis, where older age (odds ratio $=1.094, p=0.0115$ ), thicker CRT at study entry (odds ratio $=1.009, p=0.0013$ ), having ERM (odds ratio $=3.619, p=0.0256$ ) and without EZD (odds ratio $=0.127$, $p=0.0045$ ) were associated with worse final CRT (Table 3).

Comparing the two groups with or without BCVA improvements, we observed that patients having worse baseline BCVA, severe baseline NPDR status, and having ERM or EZD were associated with significant logMAR BCVA improvements in univariate analysis. Subjects with mild NPDR status were not associated with significant BCVA recovery (Table 2). However, in multivariate analysis, only having ERM (ERM with no final $\mathrm{BCVA}$ recovery, odds ratio $=0.160, p=0.0219)$ or $\mathrm{EZD}(\mathrm{EZD}$ with no final BCVA recovery, odds ratio $=0.134, p=0.0111$ ) was associated with significant final BCVA recovery.

Searching for factors predictive of frequent injections, we discovered that eyes with thicker CRT and worse BCVA at study entry were associated with more Ranibizumab injections in the univariate analysis (Table 2). Multivariate analysis confirmed that only thicker baseline CRT was predictive of receiving more than six injections (odd ratio $=1.006, p=0.011$ ) (Table 3). 


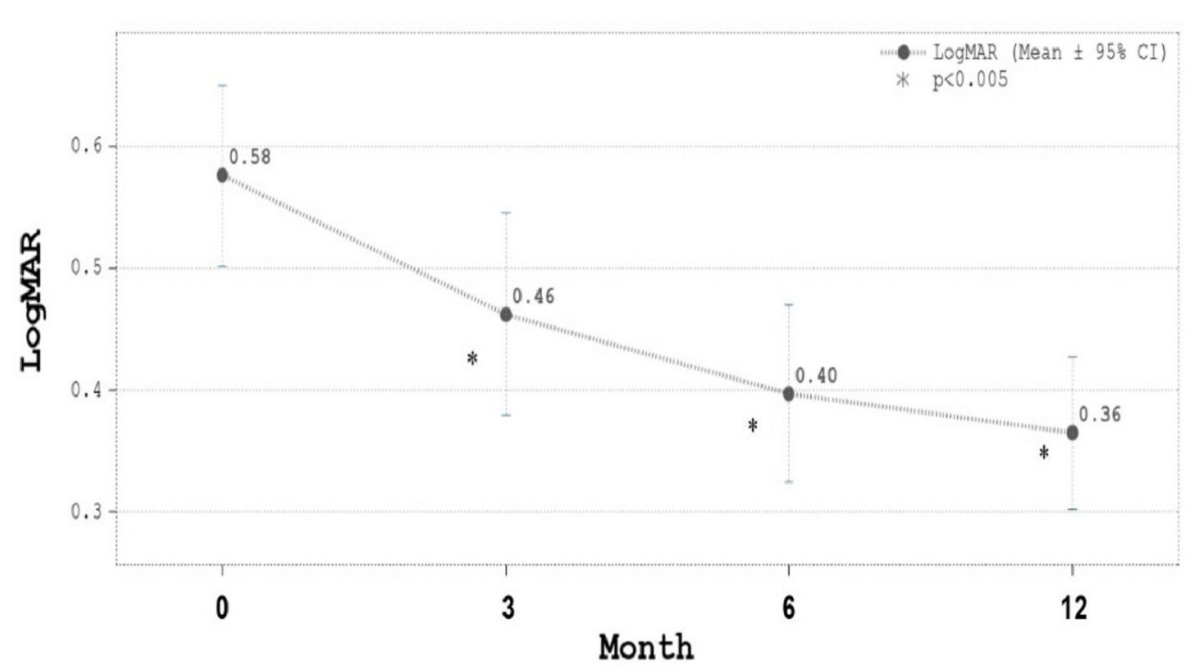

FIGURE 2 | Best-corrected visual acuity (BCVA) in LogMAR during ranibizumab treatment. LogMAR was $0.58 \pm 0.36$ (mean \pm SD) at baseline, $0.46 \pm 0.40 \mu \mathrm{m}$ at Month $3,0.40 \pm 0.35$ at Month 6, and $0.36 \pm 0.30$ at Month 12. Mean LogMAR BCVA improved significantly from 0.58 at baseline to 0.36 in month 12 .

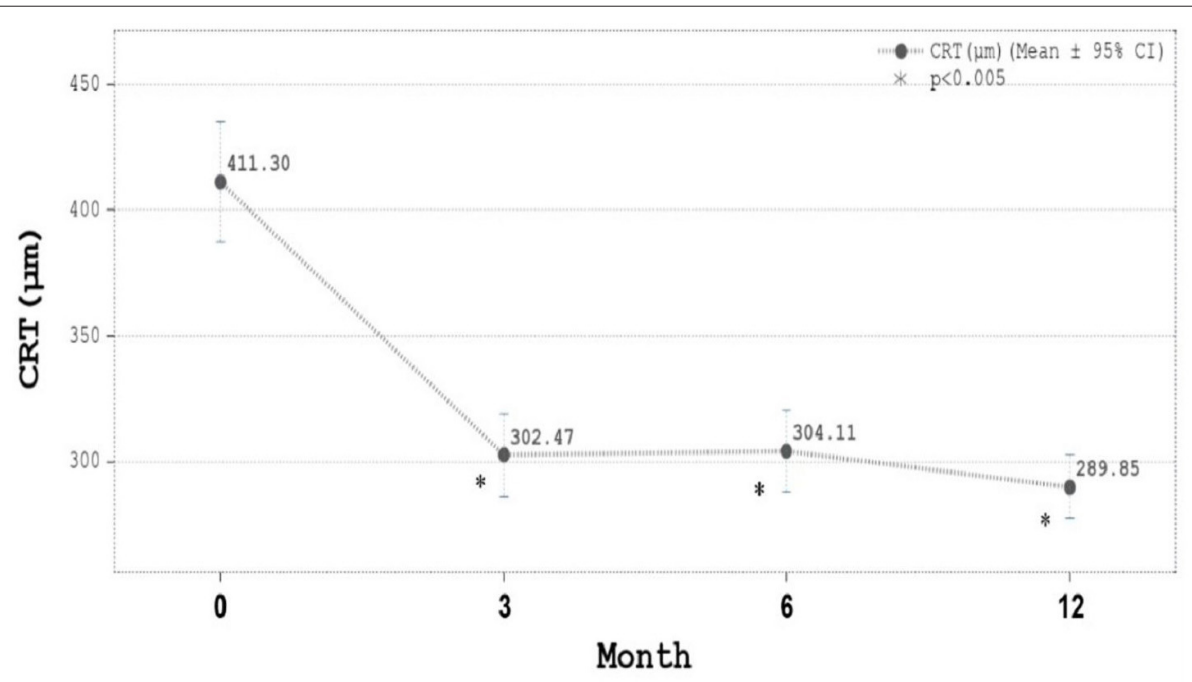

FIGURE 3 | Central retinal thickness (CRT, $\mu \mathrm{m}$ ) during ranibizumab treatment. CRT was $411.30 \pm 114.61 \mu \mathrm{m}$ (mean \pm SD) at baseline, $302.47 \pm 79.22 \mu \mathrm{m}$ at Month 3, 304.11 \pm 78.59 at Month 6, and $289.85 \pm 60.71 \mu \mathrm{m}$ at Month 12. Mean CRT decreased significantly from $411 \mu \mathrm{m}$ at baseline to $289 \mu \mathrm{m}$ in month 12 .

Among the OCT biomarkers, only the presence of EZD and ERM significantly influenced the final BCVA improvements and final CRT (Tables 2, 3). No OCT biomarkers statistically significantly influenced the total injection times in both univariate and multivariate analysis (Tables 2, 3).

\section{DISCUSSION}

We have demonstrated the efficacy of T\&E regimen with $0.5 \mathrm{mg}$ ranibizumab by 4 -week intervals. On average, the BCVA gain was 10 letters after 12 months. These results were concordant with the clinical trials that adopt extended 2-week treatment intervals. For instance, in the TREX-DME 1-year study, a standard interval of 2 weeks was adopted and BCVA gains were 9.6 and 9.5 letters for the respective T\&E and T\&E plus laser arms (16). Our study, though adopting an interval twice as long as the standard interval, attained similar BCVA gains as in the TREX-DME 1year study. Moreover, on average, only eight injections per year were required to reach efficacy, whereas the TREX-DME 1-year study required 10.7 injections (16). Hence, we demonstrated that a longer 4-week T\&E interval is feasible and economical for achieving adequate control of DME.

In the present analysis, ranibizumab reduced the average CRT by $121 \mu \mathrm{m}$ at month 12 (Figure 3). Similarly, in the TREXDME trial, CRT was reduced by 123 and $146 \mu \mathrm{m}$, respectively, for monthly and T\&E regimens. No statistical significance were 
TABLE 2 | Intercohort univariate analysis.

\begin{tabular}{|c|c|c|c|c|c|c|c|c|c|}
\hline \multirow{2}{*}{$\begin{array}{l}\text { Baseline } \\
\text { Clinical data }\end{array}$} & \multicolumn{2}{|c|}{ Grouped By final CRT } & & \multicolumn{2}{|c|}{ Grouped By final VA change } & & \multicolumn{2}{|c|}{ Grouped By injection times } & \\
\hline & $<300 \mu \mathrm{m}$ & $\geq 300 \mu \mathrm{m}$ & & Improved & Not improved & & $\leq 6$ shots & $>6$ shots & \\
\hline Age (years) & $57.64 \pm 11.66$ & $64.48 \pm 7.63$ & $p<0.05^{\dagger}$ & $60.85 \pm 9.19$ & $58.82 \pm 14.45$ & NS & $59.30 \pm 13.14$ & $61.05 \pm 8.71$ & NS \\
\hline Gender (Female) & 20/39 (51.3\%) & 9/25 (36.0\%) & NS & $21 / 47$ (44.7\%) & $8 / 17(47.1 \%)$ & NS & $12 / 27(44.4 \%)$ & $17 / 37$ (45.9\%) & NS \\
\hline $\mathrm{HbA1c}(\%)$ & $7.47 \pm 1.03$ & $7.40 \pm 1.02$ & NS & $7.47 \pm 0.92$ & $7.37 \pm 1.29$ & NS & $7.24 \pm 0.98$ & $7.59 \pm 1.04$ & NS \\
\hline $\mathrm{CRT}(\mu \mathrm{m})$ & $392.76 \pm 108.58$ & $445.47 \pm 119.24$ & $p<0.05^{\dagger}$ & $421.36 \pm 118.39$ & $384.72 \pm 101.42$ & NS & $366.58 \pm 86.84$ & $434.40 \pm 120.87$ & $p<0.05^{\dagger}$ \\
\hline LogMAR & $0.59 \pm 0.37$ & $0.55 \pm 0.33$ & NS & $0.63 \pm 0.36$ & $0.42 \pm 0.29$ & $p<0.05$ & $0.47 \pm 0.30$ & $0.63 \pm 0.37$ & $p<0.05^{\dagger}$ \\
\hline \multicolumn{10}{|l|}{$\begin{array}{l}\text { DR staging/OCT } \\
\text { Biomarkers }\end{array}$} \\
\hline Mild NPDR & $3 / 59(5.08 \%)$ & $1 / 32(3.13 \%)$ & NS & $1 / 66(1.52 \%)$ & $3 / 25(12.0 \%)$ & $p<0.05$ & $3 / 31$ (9.68\%) & $1 / 60(1.67 \%)$ & NS \\
\hline Moderate NPDR & 9/59 (15.3\%) & 5/32 (15.6\%) & NS & 11/66 (16.7\%) & 3/25 (12.0\%) & NS & 5/31 (16.1\%) & 9/60 (15.0\%) & NS \\
\hline Severe NPDR & 16/59 (27.1\%) & 12/32 (37.5\%) & NS & 25/66 (37.9\%) & $3 / 25$ (12.0\%) & $p<0.05$ & 7/31 (22.6\%) & 21/60 (35.0\%) & NS \\
\hline PDR & 3/59 (5.08\%) & 3/32 (9.38\%) & NS & 4/66 (6.06\%) & 2/25 (8.00\%) & NS & 2/31 (6.45\%) & 4/60 (6.67\%) & NS \\
\hline PDR s/p PRP & $28 / 59(47.5 \%)$ & 11/32 (34.4\%) & NS & 25/66 (37.9\%) & $14 / 25$ (56.0\%) & NS & $14 / 31$ (45.2\%) & $25 / 60$ (41.7\%) & NS \\
\hline $\operatorname{SRF}(+)$ & 14/59 (23.7\%) & 4/32 (12.5\%) & NS & 13/66 (19.7\%) & 5/25 (20.0\%) & NS & 4/31 (12.9\%) & 14/60 (23.3\%) & NS \\
\hline $\operatorname{IRC}(+)$ & 45/59 (76.3\%) & 26/32 (81.3\%) & NS & $54 / 66$ (81.8\%) & 17/25 (68.0\%) & NS & 25/31 (80.6\%) & 46/60 (76.7\%) & NS \\
\hline ERM (+) & $10 / 59(16.9 \%)$ & $13 / 32(40.6 \%)$ & $p<0.05^{\dagger}$ & 21/66 (31.8\%) & $2 / 25(8.00 \%)$ & $p<0.05^{\dagger}$ & 5/31 (16.1\%) & 18/60 (30.0\%) & NS \\
\hline EZD (+) & $21 / 59(35.6 \%)$ & $5 / 32(15.6 \%)$ & $p<0.05^{\dagger}$ & 24/66 (36.4\%) & $2 / 25(8.00 \%)$ & $p<0.05^{\dagger}$ & 7/31 (22.6\%) & 19/60 (31.7\%) & NS \\
\hline DRIL (+) & 20/59 (33.9\%) & 11/32 (34.4\%) & NS & 23/66 (34.8\%) & 8/25 (32.0\%) & NS & 9/31 (29.0\%) & 22/60 (36.7\%) & NS \\
\hline $\mathrm{HE}(+)$ & 45/59 (76.3\%) & 25/32 (78.1\%) & NS & 53/66 (80.3\%) & 17/25 (68.0\%) & NS & 23/31 (74.2\%) & $47 / 60$ (78.3\%) & NS \\
\hline
\end{tabular}

CRT, Central Retinal Thickness; DR, Diabetic Retinopathy; NPDR, Non Proliferative Diabetic Retinopathy; PDR, Proliferative Diabetic Retinopathy; PRP, Panretinal Photocoagulation; SRF, Subretinal Fluid; IRC, Intra-Retinal Cyst; ERM, Epiretinal Membrane; EZD, Ellipsoid Zone Disruption; DRIL, Disorganization of Retinal Inner Layers; HE, Hard Exudate. N: Indicates a non-statistically significant. TIndicates that statistical significance in this univariate analysis was also confirmed in multivariate analysis in Table 3. The bold values indicate $p<0.05$ 
TABLE 3 | Multiple logistic regression.

\begin{tabular}{|c|c|c|c|c|c|c|c|}
\hline Parameter & DF & Estimate & Standard Error & Wald Chi-Square & $P$-value & Odds ratio & $95 \%$ Wald \\
\hline \multicolumn{8}{|c|}{ Dependent variable: Final CRT $\geq 300 \mu \mathrm{m}$ (event) vs. Final CRT $<300 \mu \mathrm{m}$} \\
\hline Intercept & 1 & -10.0342 & 2.8891 & 12.0624 & 0.0005 & & \\
\hline Age & 1 & 0.0897 & 0.0355 & 6.3803 & 0.0115 & 1.094 & $1.020-1.173$ \\
\hline ERM $(+)$ & 1 & 0.6431 & 0.2882 & 4.9807 & 0.0256 & 3.619 & $1.170-11.20$ \\
\hline EZD (+) & 1 & -1.0323 & 0.3638 & 8.0525 & 0.0045 & 0.127 & $0.030-0.528$ \\
\hline Initial CRT & 1 & 0.00873 & 0.00272 & 10.2745 & 0.0013 & 1.009 & $1.003-1.014$ \\
\hline \multicolumn{8}{|c|}{ Dependent variable: Final VA not improved (event, $\Delta$ LogMAR $\geq 0$ ) vs. Final VA improved } \\
\hline Intercept & 1 & -2.1631 & 0.5133 & 17.7602 & $<.0001$ & & \\
\hline ERM $(+)$ & 1 & -0.9148 & 0.3991 & 5.2546 & 0.0219 & 0.160 & $0.034-0.767$ \\
\hline EZD (+) & 1 & -1.0054 & 0.3960 & 6.4451 & 0.0111 & 0.134 & $0.028-0.632$ \\
\hline \multicolumn{8}{|c|}{ Dependent variable: Injections > 6 shots (event) vs. Injection times $\leq 6$ shots } \\
\hline Intercept & 1 & -1.9051 & 0.9950 & 3.666 & 0.056 & & \\
\hline Initial CRT & 1 & 0.0065 & 0.0025 & 6.507 & 0.011 & 1.006 & 1.002-1.012 \\
\hline
\end{tabular}

Other Variables included before model selection were as following: gender, age, HbA1C, initial LogMAR, initial CRT, DR staging and initial OCT data. CRT, Central Retinal Thickness; DR, Diabetic Retinopathy; EZD, Ellipsoid Zone Disruption; ERM, Epiretinal Membrane.

observed between the two protocols in that trial (8). Interestingly, we demonstrated that the improvements of BCVA and CRT were parallel and significant starting from month 3 and until month 12 (Figures 2, 3). Moreover, the greatest increases in both BCVA and CRT were observed after 3 monthly loading injections. Protocol I from Diabetic Retinopathy Clinical Research (DRCR) and research by Lai et al. had similar results in demonstrating that $\mathrm{BCVA}$ restoration after the 3 monthly injections was predictive of long-term visual benefits $(12,17)$.

Even though we have established the efficacy of ranibizumab in DME as in other clinical trials, 35.6 and $27.5 \%$ of our patients, respectively, failed to response in CRT and BCVA under the study protocol. In both univariate and multivariate analysis, we discovered that younger age, initial CRT, and the presence of ERM or EZD were significant clinical parameters that influenced final CRT outcomes. Younger ages were, in previous studies, correlated with better vision recovery but its relation to final CRT outcomes had not been established $(10,18)$. However, the age was not significantly associated with BCVA recovery in our study. Instead, we found that age was only predictive of thinner final CRT.

Aside from age, we found that thinner baseline CRT was predictive of better final CRT while thicker baseline CRT is predictive of worse final CRT outcome. Bressler et al. proposed that a thicker baseline CRT may result in a failure to achieve the ideal final CRT of $<250 \mu \mathrm{m}$, but may still attain greater reduction of macular edema in the end (10). Hence, eyes having more severe DME with thicker CRT at the beginning may still benefit from anti-VEGF injections.

Whether baseline CRT predicts better BCVA recovery is controversial. While eyes with thinner retinal thickness is expected to have lesser capacity for BCVA improvements, baseline CRT in our study did not make a difference in final vision recovery. Contrary to our present analysis, the RESTORE trial reported that eyes with thicker initial CRT experienced greater VA gains (6). Of note, in this trial baseline BCVA of the thicker retina was not adjusted and may be a confounding factor to vision gain analysis. In later literature, Well et al. and others have demonstrated that baseline BCVA is a stronger predictor of visual improvement than retinal thickness $(19,20)$. Also, it has been observed that visual acuity may not be compatible with a given degree of macular edema. That is, one may have better gain in vision but develop a paradoxical increase in retinal thickness (21). Therefore, though it is possible that retinal thickness is modestly related to functional vision outcome, its impact may not be as essential as initial BCVA status.

Since factors other than CRT reductions relate to vision improvement, researchers have explored the microstructure of the retina in search of other co-variables $(13,22)$. The presence of photoreceptor integrity and the co-existence of vitreoretinal interface (VRI) abnormality may affect visual outcome (23). In our study, eyes with EZD had thinner final CRT compared with eyes without EZD. We hypothesized that the reduction of CRT is related to external limiting membrane (ELM) defect. ELM is considered as the organized layer that comprised of the cellular attachment between Muller glial cells and contact between Muller cells and photoreceptors $(24,25)$. In addition to the anatomical location of the ELM, the presence of tight junction proteins such as occludins on it, further support the notion that, the ELM serves as a retinal barrier between the inner retinal layer and outer photoreceptor segments. In eyes with DME, there are ELM defect due to swollen Muller glial cells and further loss of occludin proteins $(24,25)$. Though our study did not measure the continuity of the ELM on OCT, ELM disruption has frequently been associated with EZD (26). Under this premise, final CRT reduction in eyes with EZD may be related to ELM defects, which facilitate the pumping function of RPE and lead to the reduction of intra-retinal fluid. Hence, under the effect of ranibizumab, eyes with EZD would have better CRT outcome than those without EZD.

One may propose that CRT reductions in EZD are related to fovea atrophy instead of true therapeutic effects seen in DME 
patients. However, in reviewing our data, we found that the final average CRT thickness in EZD group was $289.85 \pm 60.71 \mu \mathrm{m}$ at 1 year, and only one eye with EZD had a CRT thickness of $<200 \mu \mathrm{m}$. Our CRT data of EZD group was far thicker than the criteria set for fovea atrophy, which was as either $<200$ or $150 \mu \mathrm{m}$ $(27,28)$. Hence, fovea atrophy was unlikely the contributing factor in our study. We suppose it was the therapeutic effect of ranibizumab and ELM disruption that led to thinner final CRT in the EZD group.

Vitreoretinal interface abnormality encompasses disorders such as ERM, vitreomacular adhesion (VMA), and vitreomacular traction (VMT), and affects the vision outcome of DME patients. Kulikov et al. revealed that the presence of ERM, VMT, and VMA were associated with less CRT reduction after anti-VEGF injections. However, this study did not analyze BCVA gains and was involved a 1 month period, long term outcomes uncertain (29). Others have found that anti-VEGF is still beneficial for eyes with VMA (30), while some have observed no difference in vision recovery and CRT reduction in VMA and ERM group $(13,31)$. VMT was, however, mostly indicative of poor visual outcome $(29,31)$. The traction from partial posterior vitreous detachment in VMT might lead to the distortion of inner retinal layer and thus adverse outcomes.

On the other hand, whether ERM precludes better BCVA gain is controversial. Intriguingly, we found that eyes with ERM have greater BCVA improvements than eyes without ERM. Other parameters such as initial CRT or the presence of DRIL, IRC, and SRF did not affect BCVA improvement. Similar to our observation, other studies had also found better vision recovery in eyes with ERM under anti-VEGF therapy; however, the mechanism is not well-known (32). Some proposed that in the absence of VMT and fibrovascular proliferation, ERM did not contribute to the difference to final BCVA (12). From our data, we conclude that the presence of ERM leads to at least noninferior BCVA recovery. It is difficult to ascertain whether having ERM lead to superior visual outcome, so further study with larger cohort is warranted. Therefore, in our study the presence of ERM should not preclude treatment with anti-VEGF since the presence of ERM does not hinder BCVA improvements.

Our study is limited as it is retrospective in nature. We acknowledge that there are no matching controls and confounding factors may exist in inter-cohort analysis. In intercohort analysis, parameters such as age, gender, and HbA1c were analyzed by individuals. For DR staging and OCT parameters, the data were analyzed by eyes. This difference did not influence the

\section{REFERENCES}

1. Sheu SJ, Lin WL, Kao Yang YH, Hwu CM, Cheng CL. Pay for performance program reduces treatment needed diabetic retinopathy - a nationwide matched cohort study in Taiwan. BMC Health Serv Res. (2018) 18:638. doi: 10.1186/s12913-018-3454-6

2. Massin P, Bandello F, Garweg JG, Hansen LL, Harding SP, Larsen M, et al. Safety and efficacy of ranibizumab in diabetic macular edema (RESOLVE Study): a 12-month, randomized, controlled, double-masked, multicenter phase II study. Diabetes Care. (2010) 33:2399-405. doi: 10.2337/dc10-0493 univariate analysis, but may lead to some bias in multivariable analysis. Analyzing only one eye from one person will resolve this problem but will also decrease the available data. For the current study, consideration of sample size still outweighs the existence of possible bias. Second, this study is of a relatively small sample size with short follow-up period. This might affect the statistical power in regression model analysis. However, most factors identified as significant in univariate analysis were also confirmed in multivariate regression.

Notwithstanding its limitations, our study bears significance in identifying that the presence of ERM should not preclude the individual from receiving anti-VEGF therapy. Additionally, we demonstrate how ranibizumab therapy affects retinal microstructure both anatomically and functionally.

In conclusion, the T\&E regimen in a 4-week interval with ranibizumab was a feasible and economical option for patients with DME. Parameters including age, initial CRT, and presence of ERM significantly influenced the outcome of T\&E regimen. Moreover, the presence ERM should not preclude patients from receiving anti-VEGF therapy. Further study with larger cohorts is warranted.

\section{DATA AVAILABILITY STATEMENT}

The raw data supporting the conclusions of this article will be made available by the authors, without undue reservation.

\section{ETHICS STATEMENT}

The studies involving human participants were reviewed and approved by China Medical University Hospital Institutional Review Board. The patients/participants provided their written informed consent to participate in this study.

\section{AUTHOR CONTRIBUTIONS}

C-JL, C-TL, Y-TH, and J-KW contributes to conception and design of the study. C-JL, C-TL, Y-TH, J-KW, N-YH, HSC, C-YC, and S-WW participated in data acquisition. C-JL, C-TL, C-YL, HB, N-YH, H-SC, C-YC, and S-WW analyzed and interpreted the data set. C-JL, C-TL, C-YL, HB, and N-YH drafted the manuscript. C-JL and C-TL supervised and revised the manuscript to meet academic standards. All authors had approved the integrity of the manuscript. the treatment of diabetic macular edema: a review of the current status, unmet needs, and emerging challenges. Adv Therapy. (2017) 34:1270-82. doi: 10.1007/s12325-017-0548-1

4. Nguyen QD, Brown DM, Marcus DM, Boyer DS, Patel S, Feiner L, et al. Ranibizumab for diabetic macular edema: results from 2 phase III randomized trials: RISE and RIDE. Ophthalmology. (2012) 119:789-801. doi: 10.1016/j.ophtha.2011.12.039

5. Elman MJ, Bressler NM, Qin H, Beck RW, Ferris FL, 3rd, Friedman SM, et al. Expanded 2-year follow-up of ranibizumab plus prompt or deferred laser or 
triamcinolone plus prompt laser for diabetic macular edema. Ophthalmology. (2011) 118:609-14. doi: 10.1016/j.ophtha.2010.12.033

6. Mitchell P, Bandello F, Schmidt-Erfurth U, Lang GE, Massin P, Schlingemann RO, et al. The RESTORE study: ranibizumab monotherapy or combined with laser versus laser monotherapy for diabetic macular edema. Ophthalmology. (2011) 118:615-25. doi: 10.1016/j.ophtha.2011.01.031

7. Shea AM, Curtis LH, Hammill BG, Kowalski JW, Ravelo A, Lee $\mathrm{PP}$, et al. Resource use and costs associated with diabetic macular edema in elderly persons. Arch Ophthalmol. (2008) 126:1748-54. doi: 10.1001/archopht.126.12.1748

8. Payne JF, Wykoff CC, Clark WL, Bruce BB, Boyer DS, Brown DM. Randomized trial of treat and extend ranibizumab with and without navigated laser versus monthly dosing for diabetic macular edema: TREX-DME 2-year outcomes. Am J Ophthalmol. (2019) 202:91-9. doi: 10.1016/j.ajo.2019.02.005

9. Sun JK, Jampol LM. The diabetic retinopathy clinical research network (DRCR.net) and its contributions to the treatment of diabetic retinopathy. Ophthalmic Re. (2019) 62:225-30. doi: 10.1159/000502779

10. Bressler SB, Qin H, Beck RW, Chalam KV, Kim JE, Melia M, et al. Factors associated with changes in visual acuity and central subfield thickness at 1 year after treatment for diabetic macular edema with ranibizumab. Arch Ophthalmol. (2012) 130:1153-61. doi: 10.1001/archophthalmol.2012.1107

11. Sophie R, Lu N, Campochiaro PA. Predictors of functional and anatomic outcomes in patients with diabetic macular edema treated with ranibizumab. Ophthalmology. (2015) 122:1395-401. doi: 10.1016/j.ophtha.2015.02.036

12. Lai TT, Yang CM, Yang CH, Ho TC, Hsieh YT. Treatment outcomes and predicting factors for diabetic macular edema treated with ranibizumab - Oneyear real-life results in Taiwan. J Formosan Med Association. (2019) $118(1 \mathrm{Pt}$ 1):194-202. doi: 10.1016/j.jfma.2018.03.009

13. Fickweiler W, Schauwvlieghe AME, Schlingemann RO, Maria Hooymans JM, Los LI, Verbraak FD. Predictive value of optical coherence tomographic features in the bevacizumab and ranibizumab in patients with diabetic macular edema (BRDME) study. Retina. (2018) 38:812-9. doi: 10.1097/IAE.0000000000001626

14. Chan A, Duker JS, Ko TH, Fujimoto JG, Schuman JS. Normal macular thickness measurements in healthy eyes using Stratus optical coherence tomography. Arch Ophthalmol. (2006) 124:193-8. doi: 10.1001/archopht.124.2.193

15. Campos A, Campos EJ, do Carmo A, Caramelo F, Martins J, Sousa JP, et al. Evaluation of markers of outcome in real-world treatment of diabetic macular edema. Eye Vision. (2018) 5:27. doi: 10.1186/s40662-018-0119-9

16. Payne JF, Wykoff CC, Clark WL, Bruce BB, Boyer DS, Brown DM. Randomized trial of treat and extend ranibizumab with and without navigated laser for diabetic macular edema: TREX-DME 1 year outcomes. Ophthalmology. (2017) 124:74-81. doi: 10.1016/j.ophtha.2016.09.021

17. Gonzalez VH, Campbell J, Holekamp NM, Kiss S, Loewenstein A, Augustin AJ, et al. Early and long-term responses to anti-vascular endothelial growth factor therapy in diabetic macular edema: analysis of protocol i data. Am J Ophthalmol. (2016) 172:72-9. doi: 10.1016/j.ajo.2016.09.012

18. Singh RP, Habbu K, Ehlers JP, Lansang MC, Hill L, Stoilov I. The impact of systemic factors on clinical response to ranibizumab for diabetic macular edema. Ophthalmology. (2016) 123:1581-7. doi: 10.1016/j.ophtha.2016.03.038

19. Wells JA, Glassman AR, Jampol LM, Aiello LP, Antoszyk AN, Baker $\mathrm{CW}$, et al. Association of baseline visual acuity and retinal thickness with 1-year efficacy of aflibercept, bevacizumab, and ranibizumab for diabetic macular edema. JAMA Ophthalmol. (2016) 134:127-34. doi: 10.1001/jamaophthalmol.2015.4599

20. Dugel PU, Hillenkamp J, Sivaprasad S, Vogeler J, Mousseau MC, Wenzel A, et al. Baseline visual acuity strongly predicts visual acuity gain in patients with diabetic macular edema following anti-vascular endothelial growth factor treatment across trials. Clin Ophthalmol. (2016) 10:1103-10. doi: $10.2147 /$ OPTH.S100764

21. Browning DJ, Glassman AR, Aiello LP, Beck RW, Brown DM, Fong DS, et al. Relationship between optical coherence tomography-measured central retinal thickness and visual acuity in diabetic macular edema. Ophthalmology. (2007) 114:525-36. doi: 10.1016/j.ophtha.2006.06.052

22. Al Faran A, Mousa A, Al Shamsi H, Al Gaeed A, Ghazi NG. Spectral domain optical coherence tomography predictors of visual outcome in diabetic cystoid macular edema after bevacizumab injection. Retina. (2014) 34:1208-15. doi: 10.1097/IAE.0000000000000059

23. Shin HJ, Lee SH, Chung H, Kim HC. Association between photoreceptor integrity and visual outcome in diabetic macular edema. Graefes Arch Clin Exp Ophthalmol.. (2012) 250:61-70. doi: 10.1007/s00417-011-1774-x

24. Saxena S, Akduman L, Meyer CH. External limiting membrane: retinal structural barrier in diabetic macular edema. Int J Retina Vitreous. (2021) 7:16. doi: 10.1186/s40942-021-00284-x

25. Omri S, Omri B, Savoldelli M, Jonet L, Thillaye-Goldenberg B, Thuret G, et al. The outer limiting membrane (OLM) revisited: clinical implications. Clin Ophthalmol. (2010) 4:183-95. doi: 10.2147/OPTH.S5901

26. Mohamed EHES, Nabil K, Gomaa AR, Haddad OHE, editors. External limiting membrane and ellipsoid zone integrity and presenting visual acuity in treatment-naive center involved diabetic macular edema. EC Ophthalmology. (2018) 2018:408-21.

27. Karst SG, Schuster M, Mitsch C, Meyer EL, Kundi M, Scholda C, et al. Atrophy of the central neuroretina in patients treated for diabetic macular edema. Acta Ophthalmol. (2019) 97:e1054-61. doi: 10.1111/aos.14173

28. Forooghian F, Yeh S, Faia LJ, Nussenblatt RB. Uveitic foveal atrophy: clinical features and associations. Archi Ophthalmol. (2009) 127:179-86. doi: 10.1001/archophthalmol.2008.564

29. Kulikov AN, Sosnovskii SV, Berezin RD, Maltsev DS, Oskanov DH, Gribanov NA. Vitreoretinal interface abnormalities in diabetic macular edema and effectiveness of anti-VEGF therapy: an optical coherence tomography study. Clin Ophthalmol. (2017) 11:1995-2002. doi: 10.2147/OPTH.S146019

30. Chang $\mathrm{CK}$, Cheng $\mathrm{CK}$, Peng $\mathrm{CH}$. The incidence and risk factors for the development of vitreomacular interface abnormality in diabetic macular edema treated with intravitreal injection of anti-VEGF. Eye. (2017) 31:762-70. doi: 10.1038/eye.2016.317

31. Eski Yucel O, Birinci H, Sullu Y. Outcome and Predictors for 2-Year Visual Acuity in Eyes with Diabetic Macular Edema Treated with Ranibizumab. J Ocular Pharmacol Therapeutics. (2019) 35:229-34. doi: 10.1089/jop.201 8.0082

32. Maryam AK, Tafgeh M, Mahmoud M, Pasha A, Ahad S, Khalil GF. Short term effect of intravitreal bevacizumab for diabetic macular edema associated with epiretinal membrane. Rom J Ophthalmol. (2018) 62:212-6. doi: 10.22336/rjo.2018.32

Conflict of Interest: The authors declare that the research was conducted in the absence of any commercial or financial relationships that could be construed as a potential conflict of interest.

Copyright (๑) 2021 Lai, Hsieh, Lin, Wang, Lin, Hsia, Bair, Chen, Chiu and Weng. This is an open-access article distributed under the terms of the Creative Commons Attribution License (CC BY). The use, distribution or reproduction in other forums is permitted, provided the original author(s) and the copyright owner(s) are credited and that the original publication in this journal is cited, in accordance with accepted academic practice. No use, distribution or reproduction is permitted which does not comply with these terms. 